\title{
Optimized Graph cut Color Image Segmentation Using Genetic Algorithm with Weighted Constraints (OGcut)
}

\section{A. Robert Singh, Suganya A}

\begin{abstract}
This paper proposes a Novel color image segmentation using Graph cut method by minimizing the weighted energy function. This method is applying a pair of optimal constraints namely: color constraint and gradient constraint. In the state-of-the-art methods, the background and foreground details are manually initialized and used for verifying the smoothness of the region. But in this proposed method, they are dynamically calculated from the input image. This feature of the proposed method can be used in color image segmentation where more number of unique segments exists in a single image. The genetic algorithm is applied to the graph obtained from the graph cut method. The crossover and mutation operators are applied on various subgraphs to populate the different segments.
\end{abstract}

Keywords: Graph cut, segmentation, genetic algorithm.

\section{INTRODUCTION}

Graph cut based segmentation [5] of a color image [8] needs labeling of foreground and background pixels manually. But, in recent applications like medical image processing, automatic labeling and segmentation are needed. The genetic algorithm achieves this with an objective of minimizing the energy function. In this method, two constraints namely color constraint and gradient constraints are used. These two constraints [7] are common parameters used for calculating smoothness of image segments. The color constraint determines with which group the pixel to be clustered. This constraint will automatically differentiate the foreground objects from the background. The gradient constraint defines smoothness of the boundary of the segment. The weight value of the energy function is used to maximize the clarity of the segment.

\section{The Proposed METHOD}

The image to be segmented is considered as a graph $\mathrm{G}=(\mathrm{V}$, E) where $\mathrm{V}$ is the set of vertices that represent the pixels of the image and the edge, $\mathrm{E}$ is the set of edges that represents whether the two ends of the edge are clustered into the same segment.

\subsection{Preprocessing}

Revised Manuscript Received on July 22, 2019.

* Correspondence Author

Dr. A. Robert Singh*, School of Computing, Kalasalingam Academy of Research and Education, Anand Nagar, India. Email: robertsinghbe@gmail.com

Suganya A, School of Computing, Sastra Deemed to be University, Thanjavur, India. Email: suganyarobert@gmail.com.
Initially, all the foreground and background details are labeled by background subtraction using local kernel color histogram [1]. Then the boundary details of the foreground elements are used to calculate the objective and the constraints. From the identified segments, the graph $\mathrm{G}$ with a number of subgraphs are mapped using graph cut method.

\subsection{Segmentation using Genetic Algorithm (SEG-GA)}

Given the graph $\mathrm{G}$, which has $\mathrm{N}$ sub-graphs where $\mathrm{N}$ is the number of foreground objects identified in the pre-processing. The genetic operators called selection, crossover and mutation are applied to these sub-graphs with the objective of minimal energy function as given in Eq.(1). $\min E(X)=$

$\sum_{a \in I_{1} \cup I_{2}} E_{1}\left(x_{a}\right)+w \sum_{a \in I_{3}} E_{2}\left(x_{a}\right)+\sum_{a \in \emptyset} E_{3}\left(x_{a}, x_{b}\right)$

$E_{1}\left(x_{a}\right)$ is the constraint to label pixels in foreground and background. $I_{1}$ and $I_{2}$ are the set of pixels in foreground and background respectively. $I_{3}$ is the set of unclassified pixels.

$\left\{E_{1}\left(x_{a}=1\right)=0 E_{1}\left(x_{a}=0\right)=\infty \forall a \in I_{1}\right.$

$\left\{E_{1}\left(x_{a}=1\right)=\infty E_{1}\left(x_{a}=0\right)=0 \forall a \in I_{2}\right.$

$E_{2}\left(x_{a}\right)$ is the color constraint that measures the cost for labelling the background and foreground pixels into the set $I_{3} . \mathrm{W}$ is the weight that balances $I_{1}$ and $I_{2}$. This weight value is dynamically fixed in the proposed method. $E_{3}\left(x_{a}, x_{b}\right)$ is the gradient term between two adjacent pixels a and $\mathrm{b}$.

\section{a. Automatic constraints}

There are two constraints in the proposed method are color constraint and gradient constraint. The color constraint term is given in Eq.(3).

$$
\left\{\begin{array}{l}
E_{2}\left(x_{a}=1\right)=\alpha\left(d_{a}^{I_{1}}, d_{a}^{I_{2}}\right) \\
E_{2}\left(x_{a}=0\right)=\beta\left(d_{a}^{I_{1}}, d_{a}^{I_{2}}\right)
\end{array} \forall a \in I_{3}\right.
$$

where

$$
\begin{gathered}
\alpha\left(d_{a}^{I_{1}}, d_{a}^{I_{2}}\right)=\frac{d_{a}^{I_{1}}}{d_{a}^{I_{1}}+d_{a}^{I_{2}}} \\
\beta\left(d_{a}^{I_{1}}, d_{a}^{I_{2}}\right)=\frac{d_{a}^{I_{2}}}{d_{a}^{I_{1}}+d_{a}^{I_{2}}}
\end{gathered}
$$

$d_{a}^{I_{1}}$ and $d_{a}^{I_{2}}$ represent the distance between $\mathrm{a}$ and the background distribution. The foreground and

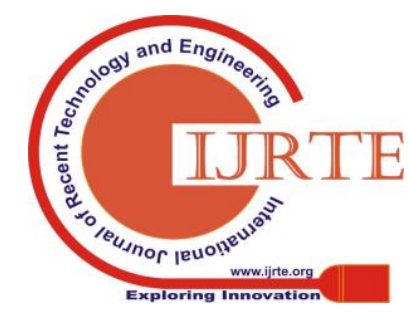


background distributions, $D^{I_{1}}$ and $D^{I_{2}}$ are already identified segments using graph cut method. Let $C_{k}^{I_{1}}$ and $C_{k}^{I_{2}}$ be the RGB values of centre points of the segment and $C_{a}^{I_{1}}$ and $C_{a}^{I_{2}}$ be the RGB values of pixel a, then the distance $d_{a}^{I_{1}}$ and $d_{a}^{I_{2}}$ are calculated as given in Eq.(4) and Eq.(5).

$$
\begin{gathered}
d_{a}^{I_{1}}=\min _{a=1,2 . . s i z e}\left(I_{1}\right)\left\|C_{a}^{I_{1}}-C_{k}^{I_{1}}\right\| \\
d_{a}^{I_{2}}=\min _{a=1,2 . . \operatorname{size}\left(I_{2}\right)}\left\|C_{a}^{I_{2}}-C_{k}^{I_{2}}\right\|
\end{gathered}
$$

The color constraint improves labeling the pixels having a similar color to the foreground or background distributions. It works well on images having different foreground and background colors. But, the pictures with similar foreground and background intensity values need gradient constraint.

As gradient gives the directional change in intensity, it is used to map the foreground and background data with overlapped color distributions. The gradient constraint $E_{3}\left(x_{a}, x_{b}\right)$ is given in Eq.(6).

$$
E_{3}\left(x_{a}, x_{b}\right)=\frac{\lambda\left|x_{a}-x_{b}\right|}{1+D_{a b}^{2}}
$$

$\lambda$ is a constant, in this work 1000 is taken. $D_{a b}$ is the difference between the RGB values of the pixels a and $b$.

The genetic algorithm [6] is applied to map the weight $\mathrm{w}$. The different subgraphs are the initial population for optimization. From the above analyses, we know that the color and gradient constraints play decisive roles in different situations. In fact, in natural or medical images, a single image is a composition of fading, and overlapped foreground and background color distributions. Therefore, different parts in a single image have different demands for the proportion of the color and gradient constraints, which is controlled by the weight $w$ in Eq.(1). The weight is chosen automatically as follows. Every time that user adds a foreground/background stroke, the foreground/background distributions are remodeled by using the graph cut method. The fitness of the proposed genetic algorithm is given in Eq.(7).
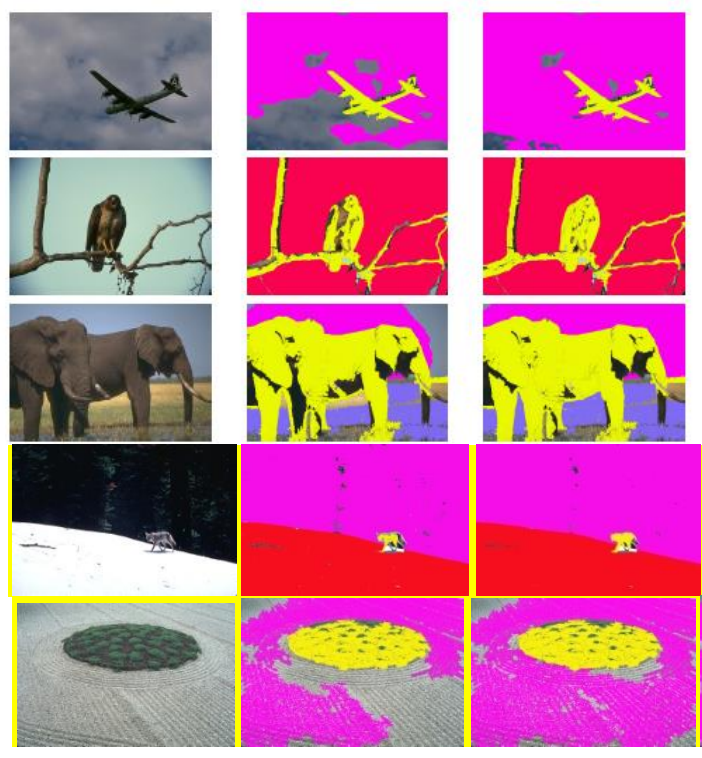

$$
f=\frac{1}{E(x)}
$$

The root of $\mathrm{G}$ is the first pixel in the image. The subgraphs represent the foreground or background segments identified earlier. Two adjacent subgraphs are selected for genetic algorithm. The objective of mapping the overlapped color segments can be outlined clearly. The random points from both subgraphs belonging to $I_{3}$ are selected for crossover. The selected pixels are exchanged and a new subgraph is obtained. Random point mutation is applied on the leaf nodes of the subgraphs to enhance the edges between two segments. The stopping criterion for genetic algorithm is getting the weight value continuously as zero for more than ten generations.

\section{ReSUlt ANALYSIS}

The proposed method is implemented using Matlab 2013b and tested for the Berkley color image segmentation data set. Figure. 1 shows the sample images from the data set and the improvement obtained by the image segmentation through different generations. The first two images have a single foreground and a common background. The airplane image reaches convergence at the generation of 38 and there is no significant improvement in the objective. The figure Eagle has similar image contents that reach convergence at generation 46. But, the figure Elephant has one foreground object and two different backgrounds say the floor and sky. The genetic algorithm converged at generation 93 and the result is shown in Figure.2. The proposed method is compared with the graph cut methods using different color models [4] like RGB, YUV, XYZ, CMY and HSV in terms of error rate and overlapping score rate. Error rate [9] is the ratio between number of pixels wrongly segmented and the total number of pixels as given in Eq.(8).

$$
\operatorname{Seg}_{\text {err }}=\frac{N_{w}}{N}
$$

where $\mathrm{N}$ is the total number of pixels of the image and $\mathrm{N}_{\mathrm{w}}$ is the number of pixels wrongly segmented.

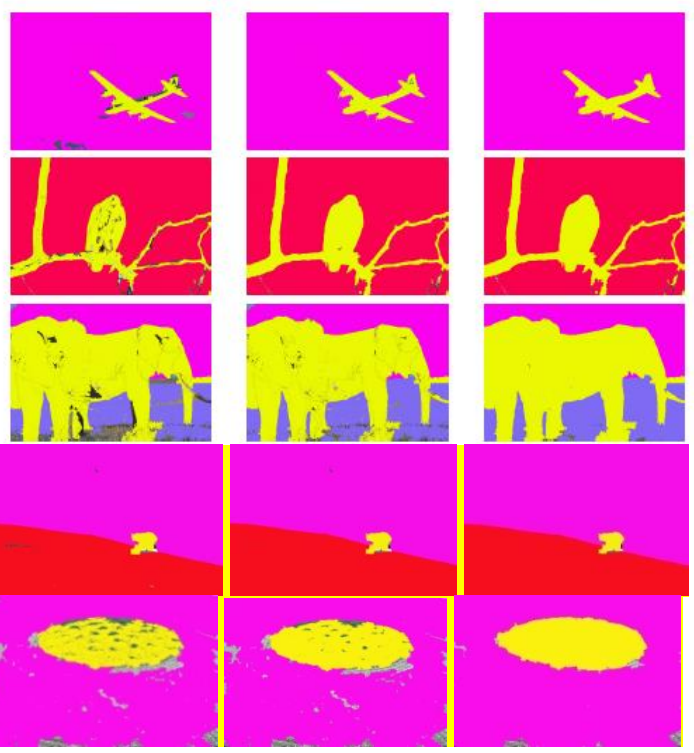


International Journal of Recent Technology and Engineering (IJRTE)

ISSN: 2277-3878, Volume-8 Issue-4S2, December 2019
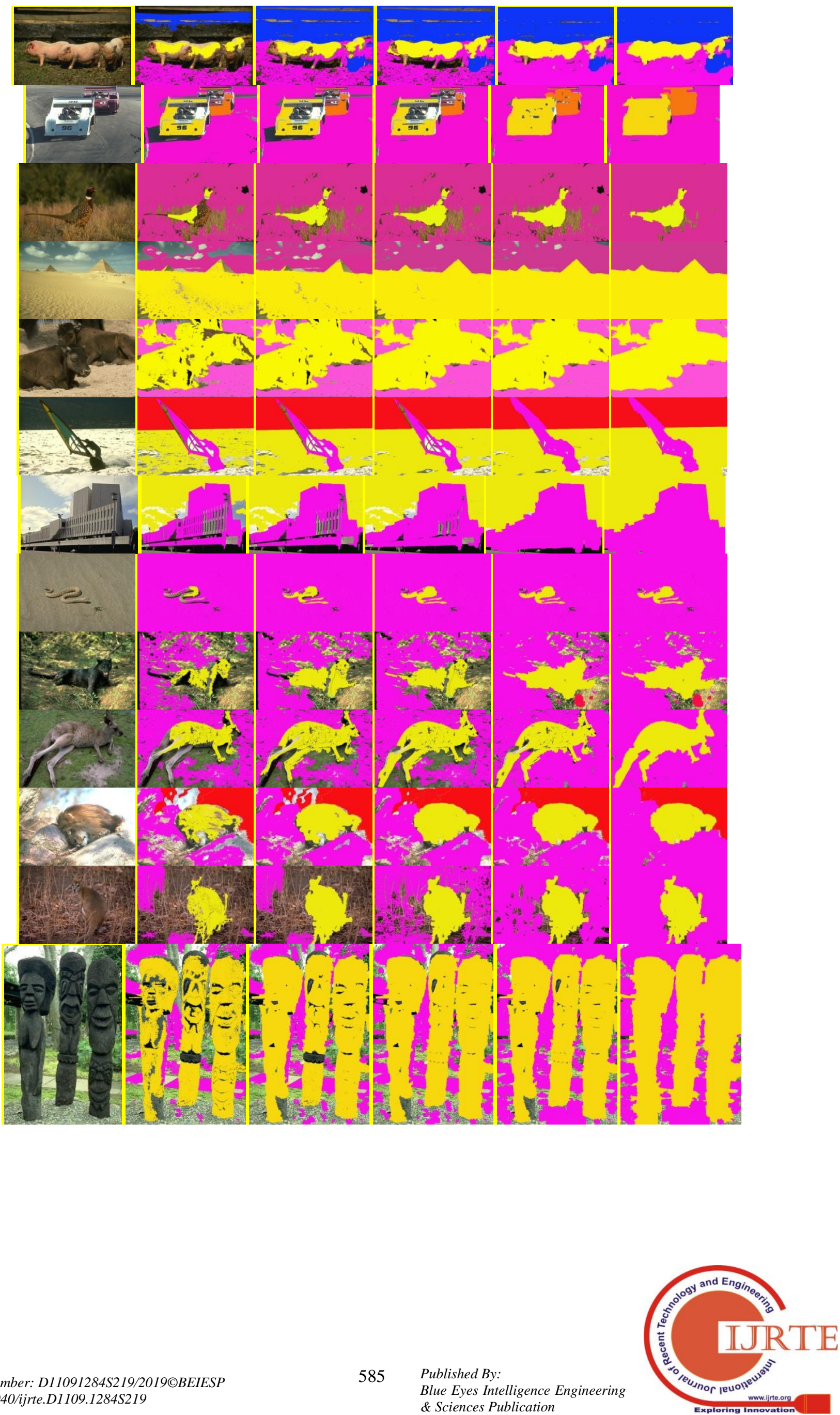

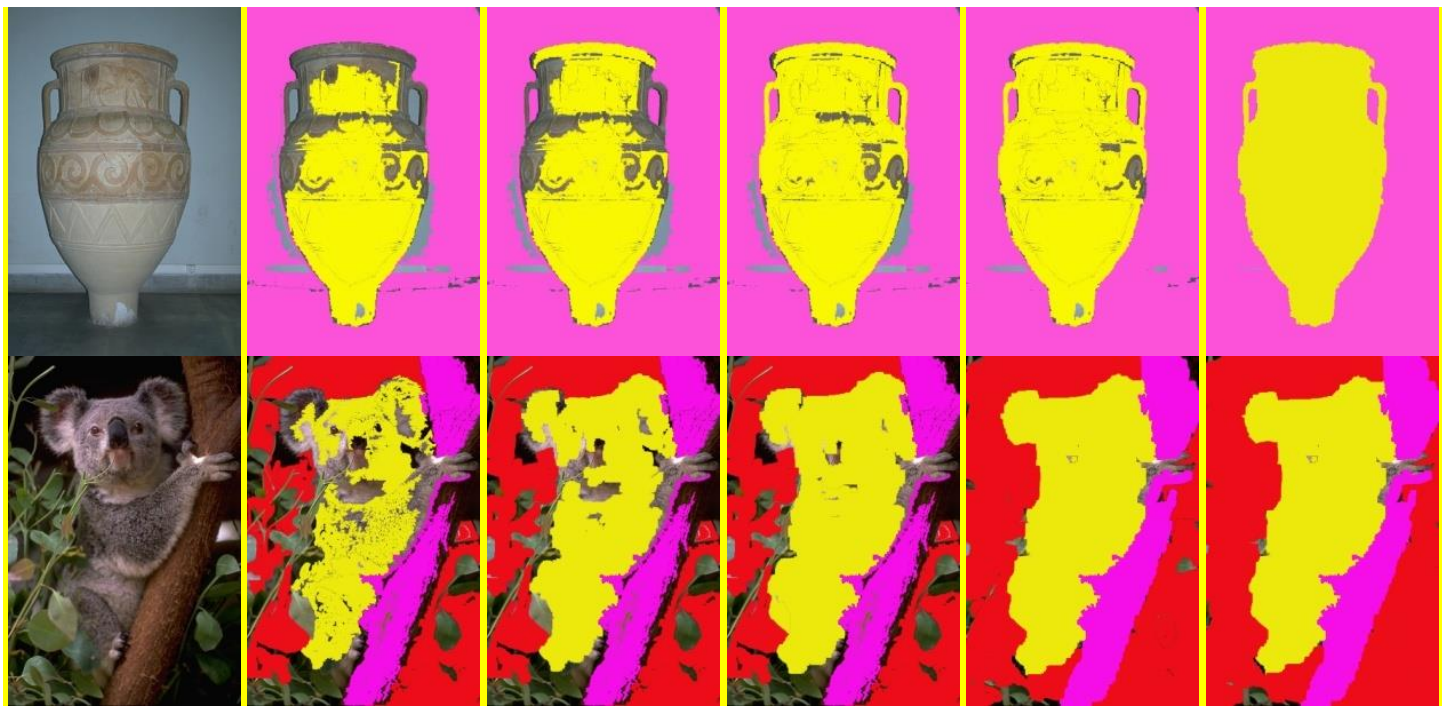

Original image Generation 10 Generation 20 Generation $30 \quad$ Generation 40 Generation 50

Fig.1. Segmentation obtained through generations 50 for Berkley color image segmentation data set [3]

The results show that the images with different foreground and background shades are segmented without overlapping. For example the figures aero plane, eagle, elephant, bush, wolf, pyramid, yacht, laying Kangaroo, stone statues and the jar has different foreground and background colors that can be easily segmented by the optimization. In particular, the wolf and yacht has three segments and all are in different intensity that leads to clear segmentation without overlapping. The remaining images like snake, the background and foreground colors are similar. In this case, the OGcut algorithm identified the segments with overlapping.

Table 1: Comparison of Error rate for different Color models and OGcut

\begin{tabular}{|c|c|c|c|c|c|c|}
\hline Image & RGB & YUV & XYZ & CMY & HSV & OGCut \\
\hline 1 & 19.53 & 20.6 & 5.56 & 37.27 & 5.85 & 4.87 \\
\hline 2 & 6.59 & 2.94 & 3.3 & 2.97 & 19.25 & 2.89 \\
\hline 3 & 7.57 & 6.31 & 4.68 & 4.2 & 5.54 & 3.4 \\
\hline 4 & 3.57 & 3.84 & 19.3 & 3.11 & 13.19 & 3.01 \\
\hline 5 & 4.17 & 3.86 & 4.82 & 43.13 & 74.71 & 3.13 \\
\hline 6 & 1.31 & 1.17 & 1.75 & 31.74 & 29.96 & 1.15 \\
\hline 7 & 3.19 & 3.33 & 3.19 & 1.86 & 37.05 & 1.69 \\
\hline 8 & 1.21 & 7.8 & 1.48 & 2.79 & 3.55 & 0.98 \\
\hline 9 & 2.77 & 3.13 & 2.65 & 31.74 & 29.36 & 2.08 \\
\hline 10 & 3.16 & 5.17 & 4.68 & 5.12 & 5.93 & 2.53 \\
\hline 11 & 2.28 & 2.18 & 2.29 & 15.16 & 16.28 & 1.49 \\
\hline 12 & 5.09 & 5.5 & 5.15 & 5.42 & 8.58 & 4.56 \\
\hline 13 & 2.9 & 3.04 & 2.83 & 43.11 & 28.24 & 2.8 \\
\hline 14 & 3.21 & 2.98 & 3.23 & 5.08 & 5.12 & 2.01 \\
\hline 15 & 4.33 & 4.3 & 4.26 & 6.26 & 13.56 & 3.42 \\
\hline 16 & 3.73 & 3.8 & 3.31 & 9.43 & 50.28 & 2.94 \\
\hline 17 & 2.34 & 2.72 & 2.35 & 36.84 & 6.44 & 1.71 \\
\hline 18 & 4.35 & 7.58 & 7.25 & 36.76 & 80.5 & 3.79 \\
\hline 19 & 3.29 & 37.34 & 45.95 & 5.6 & 6.5 & 2.7 \\
\hline 20 & 2.05 & 1.86 & 1.7 & 9.44 & 28.3 & 1.42 \\
\hline
\end{tabular}

\begin{tabular}{|r|r|r|r|r|r|r|}
\hline 21 & 1.5 & 1.92 & 1.12 & 1.67 & 22.8 & 0.18 \\
\hline 22 & 3.34 & 3.8 & 3.42 & 5.56 & 6.04 & 3.09 \\
\hline 23 & 3.34 & 3.99 & 3.95 & 3.95 & 3.99 & 3.04 \\
\hline
\end{tabular}

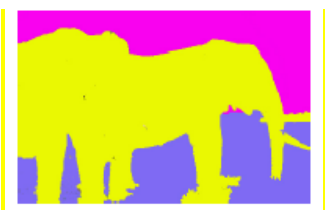

Fig.2. Convergence of Elephant at generation

Table 1. Summarizes the error rate for the images available in the Berkeley image segmentation set using the graph cut method for segmentation with different color models. The result shows that the OGcut method achieves minimum error rate than the other methods. Table 2. Compares the overlapping score rate for different images in Berkeley data set using the proposed method and the graphcut methods with different color models. The results show that the proposed method achieves a better image segmentation than other graphcut color image segmentation methods. The average accuracy measures of the graph cut segmentation with different color models and the proposed method are given in Figure. 3 for all the images in Berkeley data set. It shows that the proposed method can be used for the color image which has more number of significant objects and multiple backgrounds.

Table 2: Comparison of Overlapping score

\begin{tabular}{|c|c|c|c|c|c|c|}
\hline Image & RGB & YUV & XYZ & CMY & HSV & OGCut \\
\hline 1 & 58.57 & 92.99 & 98.47 & 98.05 & 99.24 & 56.57 \\
\hline 2 & 75.69 & 98.85 & 98.63 & 97.95 & 98.86 & 73.69 \\
\hline 3 & 85.48 & 95.38 & 99.21 & 99.31 & 98.22 & 82.48 \\
\hline 4 & 97.02 & 99.19 & 88.13 & 99.11 & 100 & 84.13 \\
\hline 5 & 85.18 & 89.33 & 85.7 & 99.19 & 96.56 & 83.18 \\
\hline 6 & 97.17 & 99.51 & 99.56 & 74.05 & 98.2 & 71.05 \\
\hline 7 & 69.01 & 99.85 & 99.76 & 93.21 & 98.43 & 68.01 \\
\hline 8 & 90.81 & 44.91 & 97.27 & & & \\
\hline
\end{tabular}




\begin{tabular}{|r|r|r|r|r|r|r|}
\hline 9 & 97.31 & 99.22 & 99.24 & 82.93 & 85.93 & 80.93 \\
\hline 10 & 96.35 & 99.9 & 99.92 & 97.39 & 100 & 94.35 \\
\hline 11 & 97.04 & 99.91 & 99.59 & 63.79 & 61.16 & 58.16 \\
\hline 12 & 89.32 & 92.48 & 93.3 & 96.81 & 81.42 & 78.42 \\
\hline 13 & 95.64 & 98.73 & 99.03 & 99.91 & 97.09 & 92.64 \\
\hline 14 & 96.41 & 98.37 & 97.97 & 94.79 & 94.55 & 92.55 \\
\hline 15 & 91.98 & 99.28 & 99.28 & 99.22 & 88.4 & 85.4 \\
\hline 16 & 93.8 & 98.89 & 98.94 & 99.56 & 99.79 & 91.8 \\
\hline 17 & 95.11 & 99.07 & 99 & 99.65 & 81.17 & 79.17 \\
\hline 18 & 91.06 & 98.95 & 98.71 & 96.36 & 100 & 90.06 \\
\hline 19 & 93.3 & 99.88 & 64.12 & 90.29 & 99.84 & 62.12 \\
\hline 20 & 96.43 & 98.95 & 99.1 & 98.04 & 98.82 & 95.43 \\
\hline 21 & 94.64 & 99.7 & 98.99 & 98.18 & 99.92 & 91.64 \\
\hline 22 & 93.73 & 94.65 & 94.36 & 89.97 & 89.85 & 85.85 \\
\hline 23 & 93.74 & 95.13 & 94.91 & 94.95 & 95.58 & 91.74 \\
\hline
\end{tabular}

International Symposium on Multimedia, Irvine, CA, 2012, pp. 487-488.

6. Amelio, Alessia \& Pizzuti, Clara. (2013). A Genetic Algorithm for Color Image Segmentation. Proceedings of the 16th European conference on Applications of Evolutionary Computation, 314-323.

7. G. Qiu, Constraint adaptive segmentation for color image coding and content-based retrieval, IEEE Fourth Workshop on Multimedia Signal Processing (Cat. No.01TH8564), Cannes, 2001, pp. 269-274.

8. Binmei Liang and Jianzhou Zhang, "KmsGC: An Unsupervised Color Image Segmentation Algorithm Based on -Means Clustering and Graph Cut," Mathematical Problems in Engineering, vol. 2014, Article ID 464875, 13 pages, 2014. https://doi.org/10.1155/2014/464875.

9. Sreenath Rao Vantaram and Eli Saber "Survey of contemporary trends in color image segmentation,"Journal of Electronic Imaging 21(4), $040901 \quad$ (4) October 2012). https://doi.org/10.1117/1.JEI.21.4.040901.

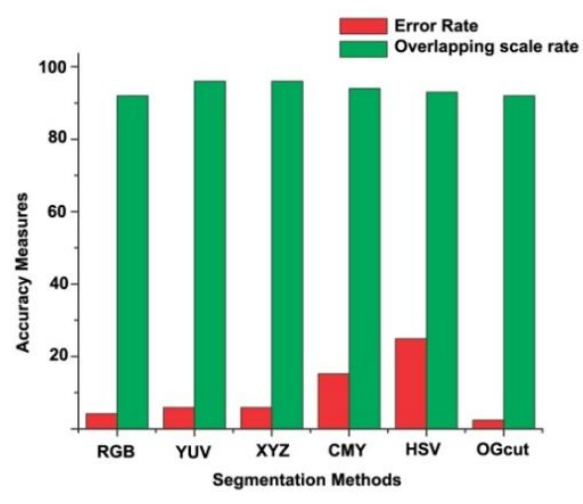

Fig. 3. Segmentation methods vs Accuracy measures (Error rate and overlap rate)

\section{CONCLUSION}

The OGcut method is an iterative optimization process that segments the color image with the two constraints that satisfy the smoothness as well as the boundary of the segments with high accuracy. The two given constraints can identify a maximum of three non-overlapping segments and to identify more segments, more constraints can be added that can improve the overlapping score.

\section{References}

1. Pedro F, Felzenszwalb Daniel P. Huttenlocher (2004).

Efficient graph based image segmentation. Comp Vision, 167-182.

2. Noriega, Philippe Bascle, Benedicte Bernier, Olivier. (2006). Local kernel color histograms for background subtration. 213-219.

3. https://www2.eecs.berkeley.edu/Research/Projects/CS/vi sion/bsds/

4. Dina Khattab, Hala Mousher Ebied, Ashraf Saad Hussein, Mohamed Fahmy Tolba. (2014).Color Image Segmentation Based on Different Color Space Models Using Automatic GrabCut. The ScientificWorld Journal, $1-10$.

5. L. Bin-mei and Z. Jian-zhou, Graph Cut Based Unsupervised Color Image Segmentation, IEEE

\section{AUTHORS PROFILE}

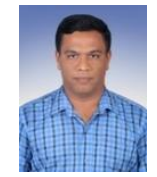

Dr. A. Robert Singh is working as an Assistan Professor in Kalasalingam Academy of Research and Education. He has completed his $\mathrm{Ph} . \mathrm{D}$ in the field of routing algorithm for AMI in smart grid.. He has completed his Ph.D in the field of routing algorithm for AMI in smart grid. He has published papers in reputed journals. His areas of interest are soft computing and Internet of Things. He is a life time member of IEI and IEEE.

Suganya A is working as an Assistant Professor in Sastra Deemed to be University. Her research areas are digital image and video processing, and Internet of Things. 